1 Running title: How migration pulsedness impacts gene flow

2 Type of article: Original Articles

3 Number of words in the main text: 6218 without abstract nor legends.

4 Number of words in the abstract: $\sim 200(\max : 200)$

5 Number of tables: 0

6 Number of figures: 5

7 Number of references: 66

\title{
Migration pulsedness alters patterns of allele fixation and local adaptation in a mainland-island model
}

\author{
Aubree, Flora ${ }^{1, *}$ \\ Lac, Baptiste ${ }^{1}$ \\ Mailleret, Ludovic ${ }^{1,2}$ \\ Calcagno, Vincent ${ }^{1}$.
}

1. Université Côte d'Azur, INRAE, CNRS, ISA, 06900 Sophia Antipolis, France.

2. Université Côte d'Azur, INRIA, INRAE, CNRS, Sorbonne Université, BIOCORE, Sophia Antipolis, France

\section{*Correspondence author. flora.aubree@inrae.fr}

Author contributions: VC and FA designed the research. BL wrote a first version of the codes and made preliminary analyses. FA conducted the research and wrote the manuscript with inputs from VC and LM.

Acknowledgement: The authors are grateful to the OPAL infrastructure from Université Côte d'Azur for providing resources and support to run stochastic simulations. They thank in particular the computation cluster NEF (Inria Sophia-Antipolis Méditerranée) and the cluster 'BPI' of ISA, Sophia-Antipolis. The authors thank Thomas Guillemaud for regular discussions and comments on the manuscript. FA was funded by a PhD fellowship from Université Côte d'Azur (IDEX "Investissements d'Avenir UCAJEDI", project reference nANR-15-IDEX-01).

Conflict of interest: The authors declare no conflict of interest.

Data accessibility statement: All simulation files and Java, $R$ and Mathematica scripts are available on Zenodo (DOI 10.5281/zenodo.4980610). 


\section{Abstract}

Gene flow, through allele migration and spread, is critical in determining patterns of population genetic structure, divergence and local adaptation. While evolutionary theory has typically envisioned gene flow as a continuous connection among populations, many processes can render it fluctuating and intermittent. We analyze mathematically a stochastic mainland-island model in continuous time, in which migration occur as recurrent "pulses". We derive simple analytical approximations regarding how migration pulsedness affects the effective migration rates across a range of selection and dominance scenarios. Predictions are validated with stochastic simulations and summarized with graphical interpretations in terms of fixation probabilities. We show that migration pulsedness can decrease or increase gene flow, respectively above or below a selection threshold that is $s \simeq-\frac{1}{N}$ for additive alleles and lower for recessive deleterious alleles. We propose that pulsedness may leave a genomic detectable signature, by differentially affecting the fixation rates of loci subjected to different selection regimes. The additional migration created by pulsedness is called a "pulsedness" load. Our results indicate that migration pulsedness, and more broadly temporally variable migration, is important to consider for evolutionary and population genetics predictions. Specifically, it would overall be detrimental to the local adaptation and persistence of small peripheral populations.

Keywords: Temporally variable migration, Effective migration rate, Stochastic simulations, Gene flow, Genomic signature, Migration load 


\section{Introduction}

Gene flow between populations, as a major determinant of evolutionary dynamics, has received a large interest since almost a century. Depending on its intensity and interactions with other evolutionary forces, gene flow has a range of contrasted effects (e.g. Felsenstein, 1976; Lenormand, 2002; Bürger, 2014; Tigano \& Friesen, 2016). In a focal population, it can enhance genetic diversity, prevent inbreeding, or on the contrary hamper local adaptation (Gomulkiewicz et al., 1999; Garant et al., 2007; Bürger \& Akerman, 2011). Across populations, it controls the spatial spread of novel mutations, the maintenance of polymorphisms, the level of population divergence and, eventually, the possibility of speciation (e.g. Maynard-Smith, 1966; Johnson et al., 2000; Yeaman \& Otto, 2011; Mailund et al., 2012; Rousset, 2013; Feder et al., 2019). These effects have been studied in a range of spatial configurations, such as simple two interconnected populations (Maynard-Smith, 1966; Yeaman \& Otto, 2011), mainland-island systems (Johnson et al., 2000; Bürger \& Akerman, 2011) or metapopulations (Slatkin, 1981; Rousset, 2013; Feder et al., 2019).

However, temporal variability in the flows of propagules is rarely questioned in theoretical studies (Peniston et al., 2019): the process of migration (here used in the sense of dispersal) is usually considered as constant, even though it is governed by time-fluctuating and potentially highly variable phenomena. As causes of temporal variability of migration rates, the most frequently cited phenomena can be classified in three categories. A first category relates to environmental variations. Geographical barriers can change depending on land bridges (Morris-Pocock et al., 2016; Keyse et al., 2018), sea levels fluctuations (Hewitt, 2000) or habitat fragmentation, (Peacock \& Smith, 1997). Oceanic or atmospheric current variations can also affect dispersal (Renner, 2004; White et al., 2010; Smith et al., 2018; Benestan et al., 2021; see also Catalano et al., 2020 for a recent attempt to quantify dispersal variability). Last, but not least, dispersal is also affected by extreme meteorological or climatic events such as floods and storms (Reed et al., 1988; Boedeltje et al., 2004) that can provoke rafting events (Garden et al., 2014; Carlton et al., 2017). These extreme phenomena are bound to become more prevalent with climate change (Masson-Delmotte et al., 2018). A second category includes variations caused by behavioral processes, such as ballooning (Bishop, 1990) or various forms of group or clump dispersal (Soubeyrand et al., 2015). And finally, a third category encompasses variations related to dispersal by animal vectors (Yamazaki et al., 2016; Martin \& Turner, 2018) or human-driven dispersal (through ballast waters for instance, see Carlton \& Cohen, 2003). All three categories of phenomena are known to result in variable dispersal rates, in the form of random fluctuations (Yamazaki et al., 2016), intermittent flows (Hewitt, 2000; Keyse et al., 2018) or episodic bursts of migration (Peacock \& Smith, 1997; Reed et al., 1988; Carlton \& Cohen, 2003; Reiners \& Driese, 2004; Morris-Pocock et al., 2016). The latter form is particularly common and is often referred to as "pulsed migration" (Boedeltje et al., 2004; Bobadilla \& Santelices, 2005; Folinsbee \& Brooks, 2007; Smith et al., 2018; Martin \& Turner, 2018).

Despite the strong evidence for temporally variable migration patterns, comparatively little has been done theoretically to explore their consequences for popula- 
tion genetics and adaptation. Nagylaki (1979); Latter \& Sved (1981) and Whitlock (1992) have all used discrete-time island models and considered neutral genetic variation only (infinite allele model). All three concur in predicting that temporal variability in migration rates should decrease effective gene flow, and thus increase differentiation among populations (see also Rousset, 2013). In a two-population model, Yamaguchi \& Iwasa (2013), and following papers, studied the fixation of incompatible mutations and the progress to allopatric speciation, and found that speciation occurred faster with variable migration. Some studies have investigated non-neutral cases in spatially heterogeneous environments, in particular in the context of source-sink population systems. Gaggiotti \& Smouse (1996) found that spacing out migration events, while keeping the mean number of migrants constant, decreased the level of genetic variation in the sink population. Rice \& Papadopoulos (2009) studied a mainland-island model and suggested that neglecting migration stochasticity would generally lead to overestimate the impacts of migration on adaptation. More recently, Peniston et al. (2019) extended the results of Gaggiotti \& Smouse (1996), questioning the impact of a temporally pulsed migration on the level of local adaptation in the sink population. They found that spacing out migration events (with a constant mean number of migrants) can either hamper or facilitate adaptation in a harsh sink population, depending on genetic scenarios. In a different context, Matias et al. (2013) studied specific diversity in a metacommunity model, and found that with randomly fluctuating migration rates, larger mean dispersal values were needed to produce the same local species richness. Overall, these studies seemingly converge on a negative impact of dispersal variability on effective migration rate. However they remain few and limited in their scope. Most of them underline the need for more attention being given to the consequences of migration variability.

The present study aims at providing a more comprehensive appraisal of the consequences of migration temporal variability, considering both neutral, beneficial and deleterious alleles, under various forms of selection and levels of dominance. We will consider a mainland-island system where a small island population receives migrants from a large mainland population (Wright, 1931; Felsenstein, 1976; Bürger, 2014). In the island population, we will model the dynamics of allele fixation in continuous time, at one locus, in diploid individuals. We will focus on the case of "pulsed" migration patterns, i.e. gene flow from the mainland occurs as bursts of migration of variable size and frequency (Rice \& Papadopoulos, 2009; Peniston et al., 2019, e.g.). We explore the range of migration "pulsedness" from continuous migration (independent migration of individuals) to very pulsed migration (rare migration of groups of individuals).

A simplified mathematical model using a low-migration limit is introduced and analyzed. We derive a simple graphical criterion that allows to predict how the level of migration pulsedness affects the effective migration rate. Predictions are validated with Monte-Carlo simulations that relax timescale separations and explicitly describe the stochastic demography of the mainland population.

We show that in the neutral case, migration pulsedness negatively impacts the effective migration rate, decreasing the rate of allele fixation. However, with selection, this conclusion can change quantitatively and even reverse. When the selection 
coefficient falls below some threshold value, we show that the qualitative impact of migration pulsedness can switch sign, so that the effective migration rate increases for deleterious alleles. This threshold is approximated as $s=-\frac{1}{N}$ for co-dominant alleles, and becomes lower for recessive deleterious alleles. Moreover, for sufficiently recessive deleterious alleles, migration pulsedness can have a non monotonous effect on the effective migration rate. The interplay of selection, dominance and drift is thus found to play an essential role in determining the effect of migration variability on effective migration rate.

Our results indicate that the effect of migration pulsedness is not uniform across loci subject to different selection regimes and dominance levels. The predictions obtained generalize several earlier theoretical results derived for specific contexts. More practically, we show migration pulsedness effectively homogenizes the fixation rate across the genome. This impacts the dynamics of genetic load and creates an additional migration load that we call the "pulsedness" load. Furthermore, migration pulsedness thus leaves a characteristic signature across the genome, in a way that could be detected using available genomic data. Overall, our results highlight migration variability as an important aspect of gene flow to be taken into account. Specifically, pulsed migration patterns would overall be less conducive to the local adaptation and persistence of small peripheral populations.

\section{Methods}

\section{MAINLAND-ISLAND MODEL}

A very large mainland population is supposed to send migrant individuals into a small island population of finite size $N$. Individuals are diploid (so that we consider $2 N$ alleles) and mate randomly within the island population. We consider an arbitrary locus at which some allele $A$ is fixed in the mainland whereas some other allele $a$ is fixed, initially, in the island population. The three possible genotypes $A A, A a$ and $a a$ have fitness values $1+s, 1+h s$ and 1 in the island population, with $h \in[0,1]$ the degree of dominance as usually defined (Thurman \& Barrett, 2016). Note that our results also apply to haploids, if one considers genic selection $(h=0.5)$ and takes the number of genes to be $N$ rather than $2 N$. For simplicity, mutation is neglected. The system is modelled in continuous time (overlapping generations, Moran, 1962), with a stochastic logistic demography that is assumed to bring back populations to their carrying capacity following a migration event.

Migration occurs as discrete migration events (pulses of migrants) corresponding to the arrival of $n$ individuals into the island population, that (transiently) takes the total population size to $N+n$ (see Yamaguchi \& Iwasa, 2013, for a similar description of migration). The overall intensity of migration is controlled by the migration rate $m$, the mean number of migrants per unit of time. We impose that the more individuals per migration event (the larger $n$ ), the less frequent the migration events, such that the mean number of migrants per unit of time $m$ stays constant. The frequency of migration events is thus $m / n$. For a given migration rate $m$, we consider increasing migration pulsedness levels, from $n=1$ to larger and less frequent packs of migrants reaching the island $(n>1)$. The case $n=1$, 


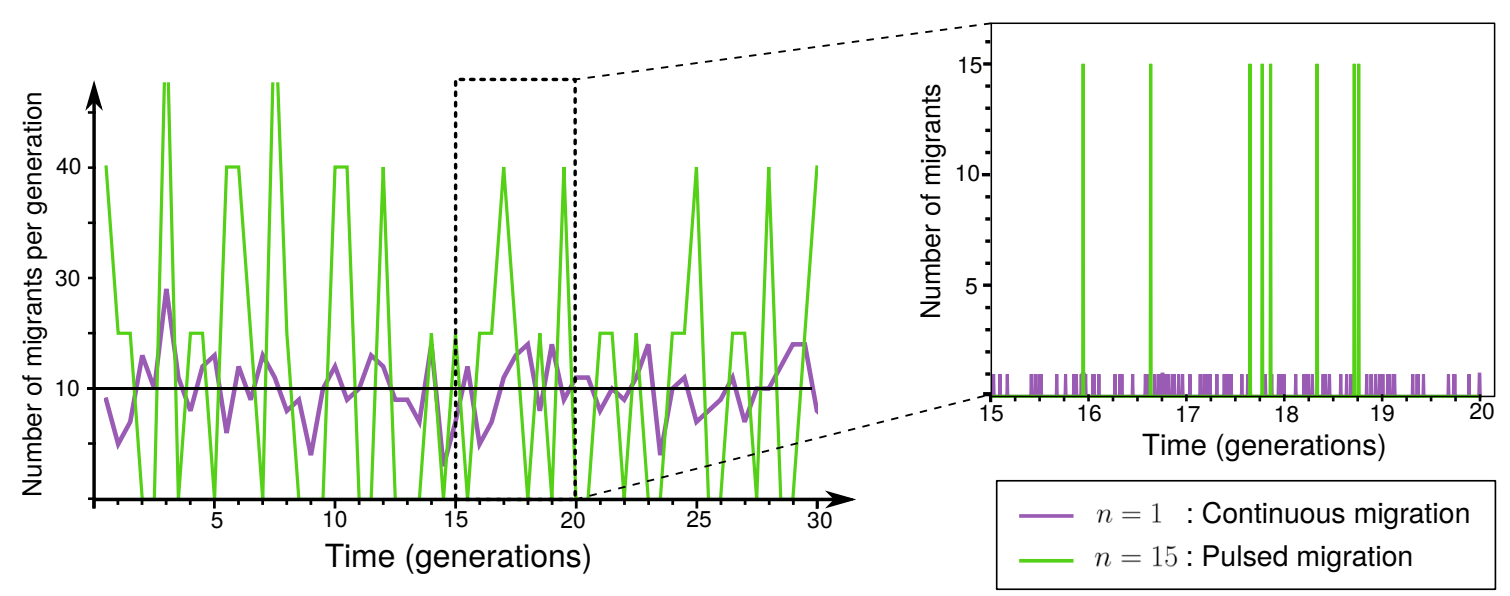

Fig. 1 Number of migrants as a function of time in the continuous case $(n=1$, independent migration of individuals) and in a pulsed migration case $(n=15)$. The left panel averages the number of migrants over 1 generation. The right panel is a close-up over 5 generations, showing the arrival of individual migrants at each migration event.

corresponding to the steady and independent migration of individuals ("continuous migration"), is taken as a reference against which the consequences of migration pulsedness will be evaluated.

The degree of migration pulsedness is thus quantified by parameter $n$. The number of migration events occurring over a period of time $T_{e}$ is assumed to follow a Poisson distribution of rate $m / n$ so that a mean of $m T_{e} / n$ migration events occur per unit time, with variance also equal to $m T_{e} / n$. The variance in the number of migrants $\left(n \times m T_{e} / n\right)$ over that period is then $n^{2} m T_{e} / n=n m T_{e}$. Parameter $n$ thus quantifies the degree of overdispersion (variance inflation) in the number of migrants per unit of time, relative to the continuous case. Under this model, migration events thus range from very frequent and of small intensity, with minimal temporal variance in the number of migrants per generation (low $n$ ), to very infrequent and intense, with large temporal variance in the number of migrants per generation (large $n$; see Fig. 1b).

To reflect the isolation of the island population and avoid regimes in which its dynamics is entirely overtaken by migration (in which case genetic homogenization is a trivial matter), the migration rate $m$ should have small enough values. More precisely, if the average generation time (here intended as the average time for $N$ selective deaths to occur, see Moran, 1962) is $T$, then $m T$ should be no greater than roughly $1-10$. This corresponds to the "one migrant per generation rule" (Mills \& Allendorf, 1996, see also Blanquart et al., 2013).

We are interested in how the mainland allele fixation rate varies with the pulsedness level $n$, across population sizes $(N)$ and selection parameters ( $s$ and $h$ ). In both the following mathematical analysis and stochastic simulations, we will derive and calculate this fixation rate. Then, to facilitate comparisons across parameter values, we will compute, in both methods too, the effective migration rate $m_{e}$, defined as the migration rate that would produce exactly the same allele fixation rate if migration was continuous (i.e. $n=1$ ). This definition is a variant of other definitions (Wang 
\& Whitlock, 2003; Kobayashi et al., 2008; Rice \& Papadopoulos, 2009) adapted to our study of migration pulsedness. The value of $m_{e}$ is $m$ when $n=1$, and deviates from $m$ for larger $n$. If, for some level of migration pulsedness, $m_{e}$ is larger (resp. lower) than $m$, we will say that the effect of pulsedness is positive (resp. negative).

The two next paragraphs describes (i) the deterministic mathematical model with it's related assumptions, and (ii) the stochastic simulations, which allow to relax some assumptions.

\section{MATHEMATICAL ANALYSIS}

To render the model analytically tractable, we make the usual assumption that demography (population regulation) is relatively fast, so that population size is effectively constant at $N$ in the island. However we cannot employ, as is also usual in population genetics, a diffusion approximation. The latter would imply to make $n$ very small relative to $N$, de facto preventing the investigation of pulsed migration patterns (see Kimura, 1962; Yamaguchi \& Iwasa, 2013). Instead, we use another approximation, by assuming that migration events are sufficiently rare $(\mathrm{m} / n$ is small enough), so that genetic drift and selection would typically result in fixation (in either direction) before the next migration event occurs. Formally, this time scale separation holds if the average time between two migration events $(n / m)$ is much larger than the average time to fixation after one migration event. From classical population genetics theory (Kimura \& Ohta, 1969; Whitlock, 2003; Otto \& Whitlock, 2013), we can show that, in the case of $n=1$ and $s=0$ (the most disfavorable case in terms of fixation time), this holds if $m \ll \frac{1}{4 N T}$ (see S.I. section S1.1). This condition is conservative: the time scale separation is more easily satisfied when $s \neq 0$ or $n>1$. We can see that this mathematical constraint is smaller than the "one migrant per generation rule" constraint introduced above $(m T<1-10)$ by a factor $4 N$ (i.e. by 2-3 orders of magnitude for the $N$ value we will consider).

Under these assumptions, at each migration event, $n$ homozygous $A A$ individuals arrive into the island population containing $N a a$ individuals. The initial frequency of the incoming allele is $f=\frac{2 n}{2 n+2 N}=\frac{n}{N+n}$ and the fast population regulation quickly takes population size back to $N$. There are two possible outcomes after such a migration event: either the mainland allele goes to fixation (the migration event is said to be a success), or it disappears and we revert to the initial state (failure) (see also Yamaguchi \& Iwasa, 2013). The mainland allele ultimately gets fixed in the island population, as soon as the first successful migration event occurs. The probability that the mainland allele has not yet gotten fixed decreases exponentially in time depending on the rate of migration events $(\mathrm{m} / n)$ and on the probability of success of each particular event. The latter is well described by the fixation probability of an allele with initial frequency $f$ in an isolated population of size $N$, written $u(f, N, s, h)$. Expressions of $u$ can be readily obtained from classical diffusion approximations (Kimura, 1962; Whitlock, 2003). To simplify notations, we write interchangeably $u(f)$ or $u(f, N, s, h)$. 


\section{Simulations}

In addition to the analysis of the mathematical model presented above, we also performed simulations to validate predictions. We simulated an island population receiving migrants from the mainland as a continuous time birth-death-immigration process, using a Gillespie algorithm. The population dynamics of the island population follows a stochastic logistic model with carrying capacity $K$ (Goel \& RichterDyn, 1974). Each diploid individual has some basal death rate $d$, birth rate $b=d$, and density dependence acts through an increase in death rate with population density (the death rate is equal to $d \frac{N^{2}}{K}$ while the birth rate is equal to $b N$ ). Selection occurs at reproduction (fertility selection). See S.I. section 1.2 for a detailed description of the model.

The island is initially fixed for allele $a$ and at carrying capacity $N=K$. At the beginning of a simulation it starts receiving $A A$ migrants from the mainland under the stochastic migration process described above. A simulation ends when allele $A$ gets fixed in the island. We conducted 10,000 replicates per parameter set to capture the stochasticity in migration times, population size, and genetic drift. To speed up simulations, we optimized the Gillespie algorithm as explained in the S.I. section 1.2. Effective migration rates were calculated from the realized fixation times using a pre-calculated abacus that returns $m_{e}$ as a function of fixation time (see S.I. section $\mathrm{S} 4$ for details and examples).

We systematically varied selection parameters $h$ (from 0 to 1 ) and $s$ (from -0.05 to 0.05 , see Thurman \& Barrett, 2016), the carrying capacity $N$ (from 50 to 200, see Palstra \& Ruzzante, 2008; Peniston et al., 2019), and migration rate $m$. Having chosen that the duration $T$ of a generation is 1 (with $d=N$, see Moran, 1962), we vary $m$ from 0.001 (that fits the mathematical assumptions), to 10 (that deviates from it by three orders of magnitude).

\section{Results}

\section{A GENERAL CRITERION TO PREDICT THE IMPACT OF MIGRATION PULSEDNESS}

Under our simplifying mathematical assumptions, the fixation rate of the mainland allele equals the product of the migration event rate $(\mathrm{m} / n)$ and the probability of success $u\left(\frac{n}{N+n}\right)$ of a particular migration event. By definition of the effective migration rate, we thus have $\frac{m_{e}}{1} u\left(\frac{1}{N+1}\right)=\frac{m}{n} u\left(\frac{n}{N+n}\right)$. It follows that $m_{e}>m$ (i.e. pulsedness has a positive effect) if and only if:

$$
u\left(\frac{n}{N+n}\right)>n u\left(\frac{1}{N+1}\right)
$$

This amounts to comparing $u\left(\frac{n}{N+n}\right)$ (the probability of fixation of $2 n$ allele copies immigrating in a single migration event) to $n$ times $u\left(\frac{1}{N+1}\right)$ (the mean number of copies that would have fixed had they arrived as $n$ independent events). In other words, the criterion determines whether the probability of fixation (through genetic drift and selection) from a group of migrants is sufficiently greater than that from a single migrant to compensate for the reduced frequency of migration events. 
a) Case $A$

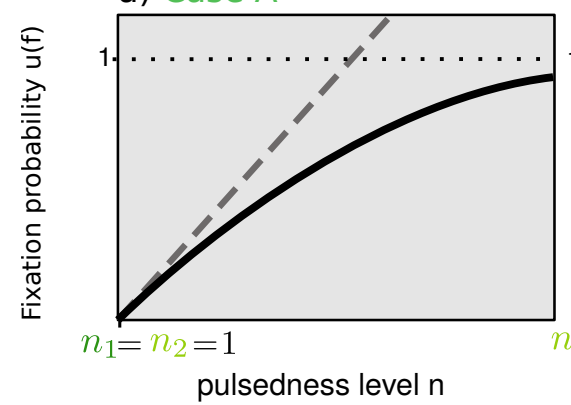

pulsedness level $n$ b) Case B

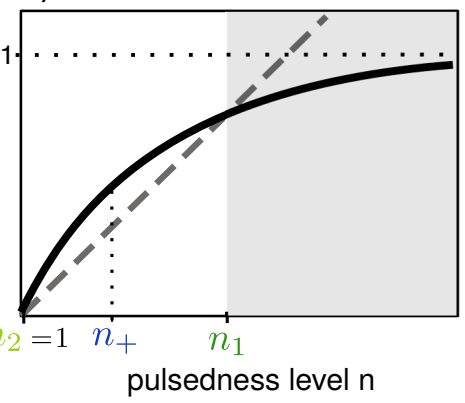

c) Case C

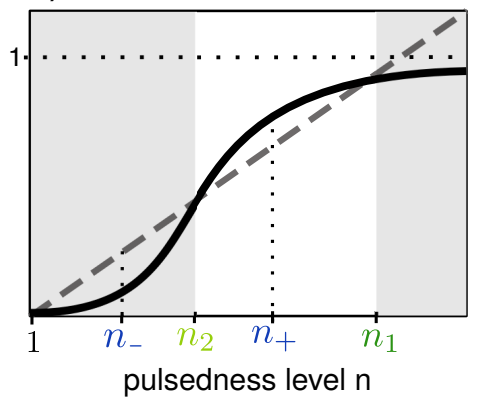

$$
\text { - - n.u }\left(\frac{1}{N+1}\right)
$$

positive effect

negative effect

Fig. 2 Graphical criterion to predict the effect of migration pulsedness (positive or negative) on the effective migration rate. The three panels represent the three possible situations. The effect is either always negative (case A) or depends on the value of $n$ (cases $\mathrm{B}$ and $\mathrm{C}$ ). $n_{1}$ and $n_{2}$ corresponds to the pulsedness values which have the same impact on fixation rate as a continuous migration. $n_{+}$(resp. $n_{-}$) corresponds to the pulsedness value which maximizes resp (minimizes) the effective migration rate. $f=\frac{n}{N+n}$.

The fixation probability $u$ is a monotonically increasing function from zero to one as $n \rightarrow \infty$ (Kimura, 1962). Compared to the linearly increasing term $n u\left(\frac{1}{N+1}\right)$ (eq. 1), it is inevitably lower for large enough values of $n$, i.e. there is some $n_{1}$ value beyond which pulsedness will always have a negative effect.

If function $u$ is strictly concave, there is no other crossing point. This is true in the absence of frequency-dependent selection or non-additivity of allelic effects in diploids (Kimura, 1962; Whitlock, 2003). Therefore there are only two possibilities: either $u\left(\frac{n}{N+n}\right)$ is always below $n u\left(\frac{1}{N+1}\right)$, and migration pulsedness always has a negative effect, i.e. it reduces the effective migration rate (case A, see fig. 2), or it can be initially greater than $n u\left(\frac{1}{N+1}\right)$, and falls below at $n=n_{1}$ (case B, see fig. 2). In that case, migration pulsedness on the contrary has a positive effect, i.e. it increases the effective migration rate, on $\left(1, n_{1}\right)$. The bifurcation from case $\mathrm{A}$ to case B occurs when the initial slope of $u(f)$ with respect to $n$ becomes greater than $u\left(\frac{1}{N+1}\right)$.

More generally, function $u$ can have an inflexion point and switch from convex to concave. This can occur when there is negative frequency-dependent selection, e.g. for recessive deleterious mutations in diploids (Kimura, 1962). In that case (case C, fig. 2), a second crossing point $n_{2}$ can exist, so that migration pulsedness can have a positive effect (increase the effective migration rate) for an intermediate range of pulsedness levels $\left(n_{2}<n<n_{1}\right)$. Bifurcations may occur from case $\mathrm{C}$ to case $\mathrm{B}\left(n_{2}\right.$ collapses into $n=1)$ or from $\mathrm{C}$ to $\mathrm{A}\left(n_{1}\right.$ and $n_{2}$ annihilate each other). This is is the entire set of possibilities.

In addition to the threshold values $n_{1}$ and $n_{2}$, one value of interest is $n_{+}$, the pulsedness level that maximizes the effective migration rate $m_{e}$ compared to $m$ (or mathematically, which maximizes the positive difference in between $u\left(\frac{n}{N+n}\right)$ and 
$n u\left(\frac{1}{N+1}\right)$ ). It is different from 1 only in cases B and C (fig. 2). Similarly, we can define $n_{-}$, a value that minimizes $m_{e}$ compared to $m$, which is different from $+\infty$ in case $\mathrm{C}$ only.

This general criterion allows to determine the consequences of migration pulsedness for any type of genetic variation, using simple graphical arguments. We will apply it to specific cases just below.

\section{NEUTRAL ALLELES}

The fixation probability for neutral alleles is known to be (Otto \& Whitlock, 2013):

$$
u(f)=f
$$

In that case, it is clear that $\frac{n}{N+n}<n \frac{1}{N+1}$ for all $n>1$. Thus, migration pulsedness should invariably decrease the effective migration rate (case A, fig. 2a). Simulations (blue points fig. 3a) confirm this mathematical prediction (for $m=0.001<\frac{1}{4 N T}$ ). Prediction holds when moving out of the mathematical limits (for $m=0.1$ ).

Intuitively, this result can be explained by greater competition among $A$ alleles in the case of pulsed migration. The rate of migration events is inversely proportional to $n$, but the initial frequency of migrant alleles (and thus the fixation probability) increases less than linearly with $n$. As a result, the former effect dominates and the effective gene flow drops as $n$ increases. The less-than-linear increase of probability of success is caused by $n$ at the denominator of $f$ : it represents the fact that with pulsed migration, packs of immigrant alleles do not only compete with resident alleles, but also among them, and more and more so as pulsedness increases. It is therefore less efficient, per capita, for alleles to arrive in a clustered way, rather than being more temporally interspersed.

\section{Alleles under SELECTION With CO-DOMinanCE}

With co-dominance $(h=1 / 2)$, or frequency-independent selection in haploids, the probability of fixation can be expressed as

$$
u(f)=\frac{1-\exp (2 N s f)}{1-\exp (2 N s)}
$$

This expression is derived from a diffusion approximation that requires large enough population sizes (Kimura, 1962; Maruyama, 1970; Whitlock, 2003).

In this case, function $u$ is concave, and criterion (1) thus follows either case A (fig. 2a) or case B (fig. 2b), depending on the selection coefficient $s$. The switch from case A (as for neutral alleles) to case B occurs for some negative value of $s$ called $s_{l 1}$. Alleles that are sufficiently selected against $\left(s<s_{l 1}\right)$ fall into case B: migration pulsedness increases the effective migration rate, at least for small levels of pulsedness (i.e. if $n<n_{1}$ ). All other cases (slightly deleterious or beneficial alleles) yield case A and the same qualitative predictions as for neutral alleles. Overall, migration pulsedness favors the fixation of deleterious alleles and it disfavors the fixation of beneficial alleles. Doing so, alleles least likely to establish (deleterious) are favored, while alleles most likely to establish (beneficial) are disfavored. This 
a)

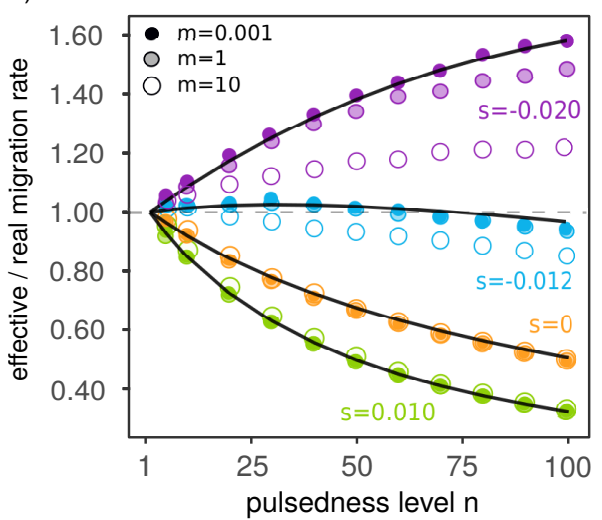

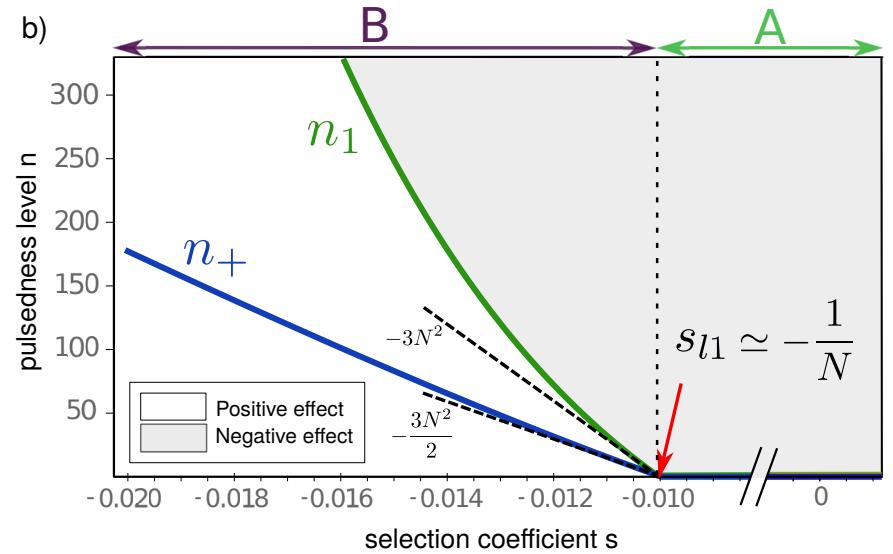

Fig. 3 Neutral and co-dominance cases. (a) Effective migration rate (relative to real migration rate $m$ ) as a function of $n$, for different selection coefficients $s$ and three migration rates. Solid lines are mathematical predictions and dots are simulated values. Here, $N=100$. The dashed line at 1 corresponds to case in which the effective migration rate is equal to the real migration rate. Above this line, the effect of migration pulsedness is positive, below it is negative. (b) Summary of curves for any $s$. The green solid line represents the theoretically expected $n_{1}$ curve as a function of $s$, and the blue one shows $n_{+}$. Dashed lines indicate the slopes of $n_{1}$ and $n_{+}$at $s=s_{l 1}$. Note that the slopes are pretty high (this figure zooms around $s=-1 / N)$, to the order of 100,000 . Here, $N=100$.

results in a homogenisation of responses across alleles which carry different selection coefficient.

Through a local approximation around $n=1$ and $s=0$ (see S.I. section S3 for details), we can show that $s_{l 1} \approx-\frac{1}{N}$ (fig. $3 \mathrm{~b}$ ). This indicates that counter selection must be strong enough relative to drift for a positive effect of migration pulsedness to arise (see also Wright, 1931; Lande, 1994). Furthermore, close to $s_{l 1}, n_{1} \approx 2 n_{+}$(see S.I. section S3) and both slopes are pretty steep, of the order of $N^{2}$ (fig. 3b). The transition is very sharp, and the value of $n_{1}$ quickly becomes very high. This means that case $\mathrm{B}$ will in practice correspond to a positive effect of migration pulsedness for alleles with $s<s_{l 1}$, since the values of pulsedness required for the effect to revert to negative are most of the time unrealistically elevated ( $n=100$ or more; see fig. $3 \mathrm{~b})$.

These predictions were verified in stochastic simulations (fig. 3a). Simulations made close to the mathematical limit $(m=0.001)$ show very good quantitative agreement (see also S.I. section S6.2), which remains rather good even 3 orders of magnitude away from it $(m=1)$. It is only when reaching the "one migrant per generation" limit $(m=10)$ that quantitative deviations appear, especially for negative $s$ values. As $m$ increases beyond our mathematical assumptions, positive effects of pulsedness are weakened or shifted to more negative $s$ values (fig. 3a). In all cases, mathematical predictions are qualitatively well supported.

An increase in population size influences the above predictions in two ways: by making $s_{l 1}$ closer to zero, and by increasing the slopes of $n_{1}$ and $n_{+}$(fig. $3 \mathrm{~b}$ ). Therefore, larger population sizes make positive effects of migration pulsedness more likely, all else equal. Approximately, figure 3 becomes almost invariant to population 


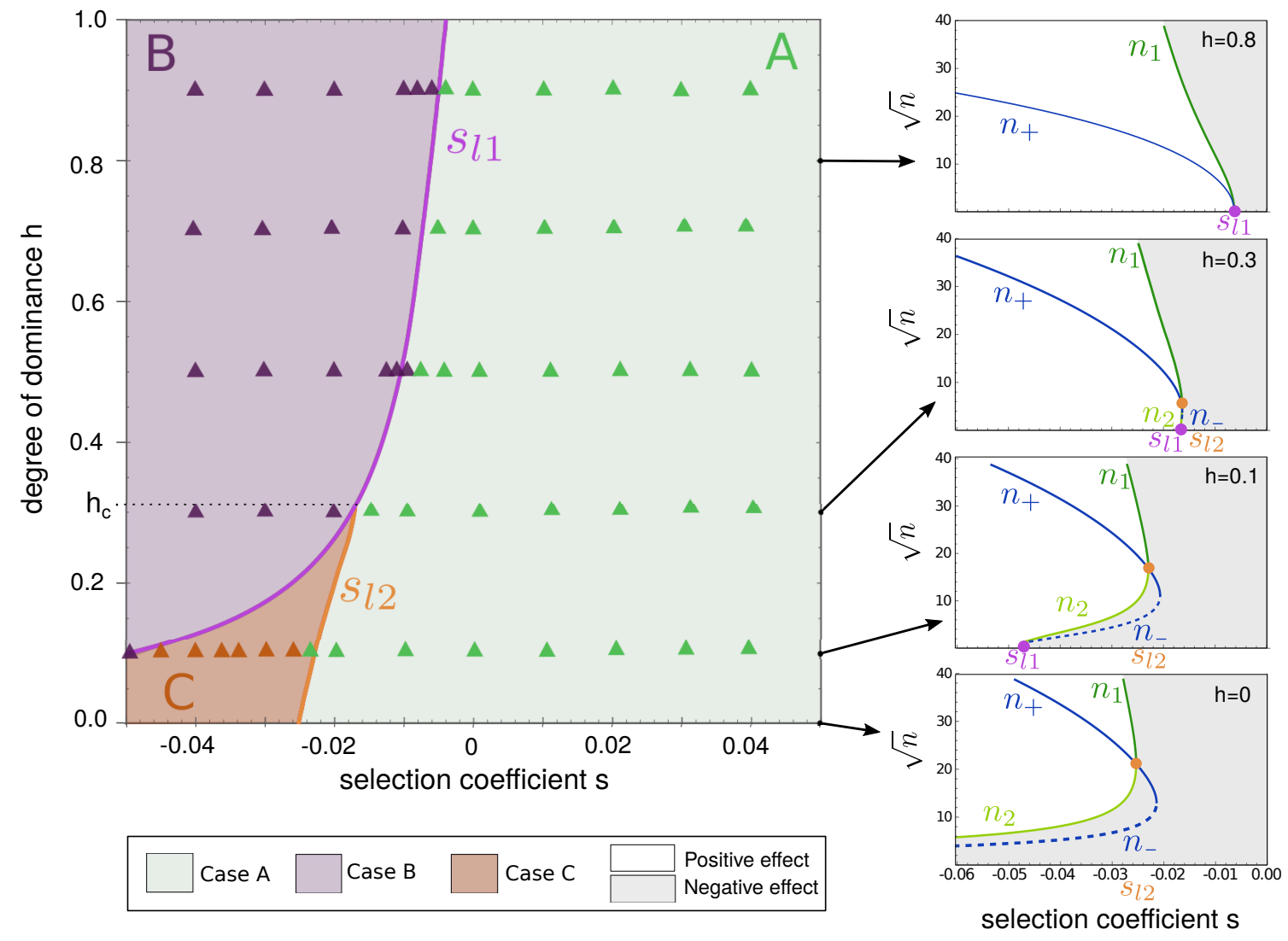

size if rescaling both axes by $N$, i.e. in terms of $n / N$ and $N s$ (see S.I. fig. S4). This indicates that predictions are largely controlled by the relative level of migration pulsedness $n / N$ and the balance between selection and drift ( $N s$; Wright, 1931; Lande, 1994).

\section{INTRODUCING DOMINANT AND RECESSIVE ALLELES}

An expression for function $u$ in the general case of any dominance is

$$
u(f)=\frac{\int_{0}^{f} G(x) d x}{\int_{0}^{1} G(x) d x}
$$

with $G(x)=\exp \left[2 N s\left((2 h-1) x^{2}-2 h x\right)\right]$ (Kimura, 1962).

Fig. 4 The effect of migration pulsedness as a function of selection coefficient $s$ and dominance $h$. The plane is partitioned according to the pattern (case A, B or C) predicted by the mathematical model. Details can be found in S.I. section S2. Simulation results are shown as triangles whose color corresponds to the observed pattern. Subpanels are cross-sections of the left panel for four specific values of dominance $(h=0.8, h=0.3, h=0.1$ and $h=0)$, similar to figure 3b. Note the square-root $y$-scale in the subpanels. 
main quantitative difference is that the value of $s_{l 1}$ gets closer to 0 as $h$ increases $\left(s_{l 1}\right.$ depends on $\left.h\right)$ : it is therefore slightly more likely to have a positive effect of migration pulsedness for dominant deleterious alleles than for recessive deleterious alleles.

In contrast, predictions differ qualitatively if $h$ moves below $h_{c}$ : moving from positive to negative $s$ values, we first have a bifurcation from case A to case $\mathrm{C}$ (at another threshold value $s_{l 2}$, that also depends on $h$ ), and then a bifurcation from case $\mathrm{C}$ to case $\mathrm{B}$ (at $s_{l 1}$ ). The positive effects of migration pulsedness first appears (at $\left.s_{l 2}\right)$ for intermediate values of $n(\mathrm{~A} \rightarrow \mathrm{C}$ bifurcation; orange line in fig.4) instead of low $n$, i.e. it requires a minimal level of pulsedness to occur. When $s<s_{l 1}$ however (after the $\mathrm{C} \rightarrow \mathrm{B}$ bifurcation; purple line in fig.4), the positive effect occurs for any $n$, as was the case for less recessive alleles. For deleterious mutations that are fully recessive $(h=0)$, case $\mathrm{C}$ is the rule (when $s<s_{l 2}$ ). However, as the minimal level of pulsedness required to get a positive effect is quite large $\left(n_{2}=40-100\right)$ in most cases, the effect would always be negative in any practical sense. Overall, when alleles are more recessive, positive effects occur under more restricted conditions (greater pulsedness levels and/or stronger counter-selection).

\section{MEAN Fitness AND PULSEDNESS LOAD}

An overarching result is that migration pulsedness homogenises fixation over loci subjected to different types of selection (see previous section), and this has important consequences at the level of entire genomes. In particular, it impacts the dynamics of genetic load. Genetic load at time $t$ is defined as the relative difference in between the maximum fitness value theoretically reachable and the mean absolute fitness actually obtained at this time (see S.I. section 7.2 for more details). Once an island population is subjected to gene flow from the mainland, beneficial alleles establish first (increasing the fitness), followed by neutral alleles, and eventually deleterious alleles. To illustrate this, we consider a large number of independent (unlinked) loci in the genome, whose selection coefficients follow a normal distribution centered on $s=0$. As expected, we observe that the genetic load initially declines and eventually goes up again (fig. 5a). This dynamics will differ depending on the level of migration pulsedness, because the latter affects allele fixation rate in different ways depending on $s$. As migration is more pulsed, the initial decline of genetic load is slower, the minimal load value is larger and its occurrence is delayed in time, and the eventual increase is also slower (fig. 5a).

As a consequence, the level of genetic load at any time depends on the level of migration pulsedness. This results in a "pulsedness load" (fig. 5b), i.e. an additional component of genetic load brought-up by the pulsed nature of migration (the difference in between the genetic load in the pulsed and in the continuous cases). This pulsedness load may account for a non negligible part of the total load ( $>10 \%$ in the example of figure 5). Variations of the pulsedness load will of course depend on the distribution of allelic effects among migrants (see fig. S6 in S.I.). The positive part of pulsedness load will be most important if migrants carry a large proportion of either beneficial (for which $n$ decreases $m_{e}$, see fig.4) or relatively strongly deleterious mutations (for which $n$ increases $m_{e}$, see fig.4). Thus, in most cases, migration pulsedness would promote maladaptation and counteract 


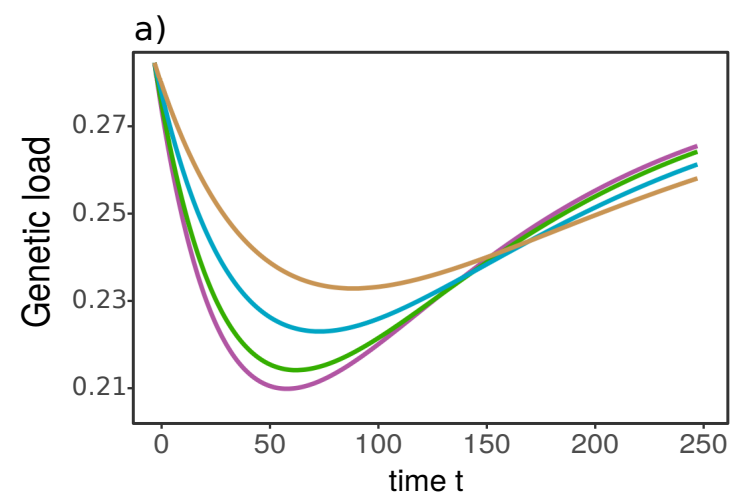

b)

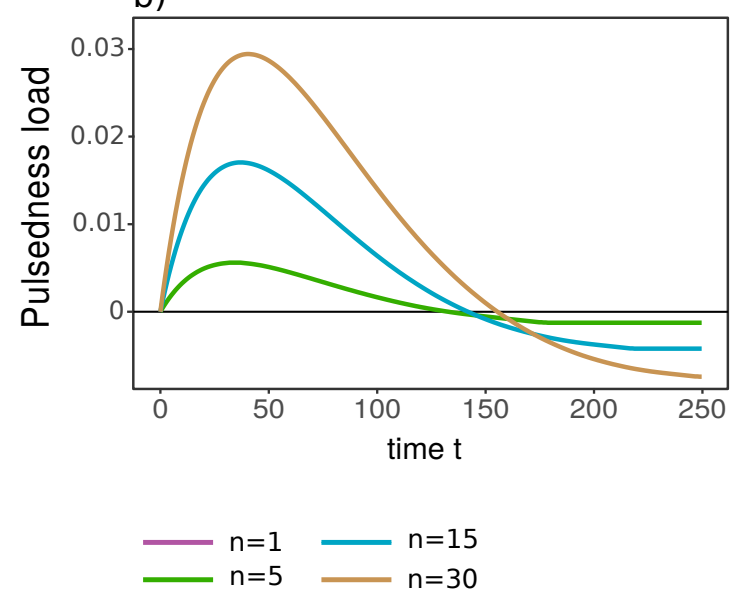

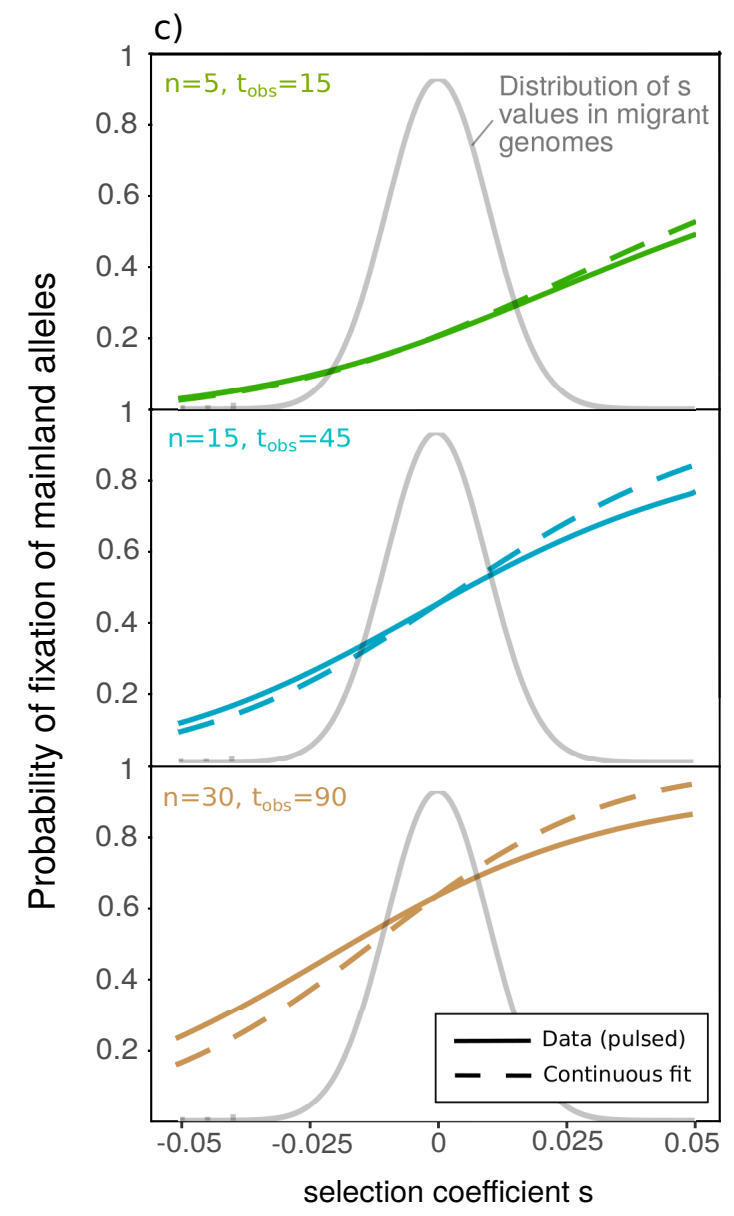

Fig. 5 Genetic load (a) and pulsedness load (b) in the island population, as a function of time after onset of migration, for different levels of migration pulsedness (n). 100 unlinked loci were considered throughout the genome, with a distribution of $s$ values normal, centered on 0 , and with standard deviation 0.01 (grey curve in (c)). Pulsedness load is first positive, when mostly non maladapted alleles fixate, then negative, when mostly maladapted alleles are left to fixate, and eventually tends to zero when all mainland alleles have fixed. (c) Genomic signature of pulsedness. Continuous fits (assuming $n=1$; dashed lines) of synthetic datasets (probabilities of fixation of mainland alleles), as a function of $s$. Synthetic datasets were generated from the mathematical model (i.e. in the limit $m \rightarrow 0$ ), for the same distribution of $s$ values (grey curve) as used in (a) and (b), and for three pulsedness levels (5, 15 and 90). Population size was $N=60$ and the time of observation $t_{\text {obs }}$ was set so that three migration events or more have occurred on average in each case. More (or less) dramatic differences can be observed for other choices of $t_{\text {obs }}$. Population size $N$ and elapsed time $t_{\text {obs }}$ were treated as unknown and estimated by maximum likelihood (see S.I. section $\mathrm{S} 8$ for details). The best continuous fits returned estimated parameters $N^{\prime}=60$ and $t_{o b s}^{\prime}=14.2$ (for $n=5$ ), $N^{\prime}=59$ and $t_{o b s}^{\prime}=35.7$ (for $n=15$ ), and $N^{\prime}=57$ and $t_{\text {obs }}^{\prime}=58.2$ (for $\left.n=30\right)$. The best continuous fit 
still pulse-disadvantaged alleles $\left(s_{l 1}<s<0\right)$ fixate. See figure S6 in S.I. for other examples with different mainland allele selection coefficients distribution.

\section{The GENOMIC SIGNATURE OF PULSEDNESS}

As migration pulsedness has a negative effect on the effective migration rate for all neutral and beneficial alleles (fig. 4), it would typically lower the overall genomic migration rate. Obviously, if one has independent estimates of the migration rate $(m)$, it would be rather straightforward to detect migration pulsedness by comparing observed fixation rates with those predicted under continuous migration. However, it is notoriously difficult to estimate average migration intensity, and such information is most often lacking (Wang \& Whitlock, 2003). Even so, the inhomogeneous impact of migration pulsedness across loci might leave a distinctive fingerprint in the genome (a genomic signature), compared to continuous migration regimes. To detect this, one would need genomic data to assess the fixation status (or frequency) of mainland alleles in the island population, at many independent loci, at some point in time. In addition, one should know, or have an indication of, the type of selection that those loci experience (ideally, a range of $h$ and $s$ values). One could then compare the fixation rates across loci in order to detect the homogenizing effect that migration pulsedness introduces. This can be achieved by fitting the observed frequencies with a model that assumes continuous migration, and test whether the data deviate from the model in the predicted direction.

The principle of this approach is illustrated in Figure 5c). We assumed a Gaussian distribution of selection coefficients of mainland alleles, centered on $s=0$, across 100 independent biallelic loci in the genome. For simplicity, all alleles were assumed co-dominant $(h=0.5)$ and the mathematical model was used. We generated artificial datasets (probabilities of fixation at each locus; see S.I. section S7.1) under different levels of migration pulsedness, and fitted these data with the continuous $(n=1)$ model by maximum likelihood (see S.I. section S8). Unknown parameters were: population size $N$, migration rate $m$, and time since onset of migration $t_{o b s}$ (see S.I. section S8 for details).

As shown in Figure $5 \mathrm{c}$, for low levels of migration pulsedness $(n=5)$, simulated datasets can be fitted quite well with a continuous migration model. However, as migration gets more pulsed ( $n=15$ or 30 ), the best continuous fit obviously deviates from the simulated data (in addition to yielding biased parameter estimates for migration rate and time since onset of migration; Figure 5). Specifically, it overestimates the fixation of beneficial alleles and underestimates the fixation of deleterious alleles, as predicted (fig. 5). It might therefore be possible to construct an operational statistical test that exploits this signal to detect migration pulsedness from genomic snapshots.

\section{Discussion}

Temporal variability in migration is likely pervasive in nature. Plants, fungi, seashells, birds and marine invertebrates are most represented in the literature about variable dispersal, but there is no reason that other taxa would not be concerned, 
including hominoids (Folinsbee \& Brooks, 2007). Yet classical evolutionary theory largely rests on the assumption of constant migration rates (Johnson et al., 2000; Yeaman \& Otto, 2011; Mailund et al., 2012; Rousset, 2013; Peniston et al., 2019, e.g.). Considering the growing evidence for non-constant migration processes, it is important to gain a general theoretical understanding of their evolutionary consequences.

We here focused on pulsed migration patterns (Yamaguchi \& Iwasa, 2013; Peniston et al., 2019). Albeit a specific form of migration variability, it is arguably quite common, and it allowed us to make significant analytical progress. Furthermore, given our stochastic modelling framework, that makes migration pulses randomly distributed in time, and based on our mathematical results, we can reasonably expect our predictions to apply more broadly to other forms of "variable" migration patterns, intended as a temporal overdispersion in the number of migrants per generation.

Confirming this, our predictions for neutral alleles are consistent with the few earlier population genetics analyses, that used modelling approaches entirely different from ours (Nagylaki, 1979; Latter \& Sved, 1981; Whitlock, 1992; Rousset, 2013). Indeed, for neutral variation, we found higher levels of migration pulsedness to decrease the effective migration rate, all else equal. In our model, the interpretation is that a larger initial frequency of immigrant alleles less-than-compensates for the accompanying lower frequency of migration events, so that the overall effect of pulsedness on fixation rate is negative. This is because migrant alleles arriving clustered in time compete more among themselves, compared to when arriving more evenly spaced out in time.

Unlike earlier analyses though, our approach could address arbitrary forms of selection, not just neutral alleles. Importantly, predictions were found to depend quantitatively, but also qualitatively, on the type of selection. The qualitative prediction that migration pulsedness negatively impacts gene-flow remains true for beneficial alleles, even though the effect size increases with selection, and for slightly deleterious alleles. This means that, for those selection scenarios, we overestimate the consequences of migration if we omit to consider its variability (see also Rice $\&$ Papadopoulos, 2009). However, for deleterious alleles, the effect weakens and at some point switches sign. For sufficiently strong counter-selection (below $s \approx-1 / N$ for additive alleles, a value reminiscent of the selection-drift balance; Wright, 1931), the effect of migration pulsedness switches to positive: pulsedness on the contrary increases the effective gene flow. This prediction radically differs from predictions based on neutral alleles.

Results with selection can be understood by the contribution of migrant alleles to the mean fitness at their locus. Compared to neutral alleles, beneficial alleles arriving in a more clustered way increase the mean fitness, thereby reducing their per-capita advantage, which further reduces the effective gene-flow. In contrast, deleterious alleles arriving in a pulsed manner lower the mean fitness, thereby alleviating their per-capita selective disadvantage. For sufficiently counter-selected alleles, this positive effect of lowering the mean fitness exceeds the negative effect of competing with identical alleles, switching the overall effect of the fixation probability to positive. 
Selection was not the only genetic factor determining the impact of migration pulsedness. The dominance level $(h)$ was also found to impact the results, in qualitative terms. Results are qualitatively similar for dominant alleles, with a slight tendency of positive effects of pulsedness to be more prevalent as $h$ increases. In contrast, results for recessive alleles, especially below $h=0.3$, differ significantly. In quantitative terms, stronger selection values are required to obtain the same results as for co-dominant alleles, presumably due to the fact that recessive alleles are 'hidden' in heterozygotes at low frequencies. Moreover, there is a qualitative difference for recessive deleterious alleles: positive effects of migration pulsedness may also require migration to be sufficiently pulsed. In other words, migration pulsedness has a non monotonous effect of gene-flow for those alleles, and there exists a minimum level of pulsedness above which the effect switches from negative to positive. This result echoes those of Peniston et al. (2019), who found that pulsedness levels were favorable to local adaptation in some intermediate pulsedness scenarios, in particular when considering density-dependent selection. It is interesting to note that the threshold value of dominance we found $(h=0.3)$ is close to the average value of dominance for mildly deleterious mutations in data and theory, as reported by Manna et al. (2011).

Our mathematical results were derived from a low-migration limit and with constant population size, but were found to hold in stochastic simulations with not-solow migration and population size fluctuations. In general, increasing the overall migration rate shifted the predictions toward a negative effect of migration pulsedness on gene-flow (Fig. 3). This is presumably caused by the direct contribution of migration to fixation in those cases (a "mass effect"), that increases the relative importance of selection/drift, and is unaffected by pulsedness. This mass effect, i.e. the fact that migrant alleles persist and accumulate over consecutive migration events, can indeed explain the observed shift towards larger values of $N s$. Mathematically speaking, an increase in initial frequency of migrant alleles due to the mass effect is equivalent to a decrease in population size (see S.I. section S6.1). It thus requires higher values of $N$ (or $s$ ) to get the same effects of migration pulsedness (see S.I fig. S4).

From a whole-genome perspective, the overarching result is that migration variability homogenizes the effective migration rate across alleles with different selection coefficients: it increases it for counter-selected alleles, and reduces it for positively selected alleles. As a consequence, throughout the genome, the fixation of beneficial variation will be slowed down, while the fixation of deleterious and maladaptive variation will be promoted. Obviously, the net impact on average population fitness will generally be negative, with higher levels of genetic load. We call this additional component of genetic load brought-up by the pulsed nature of migration the "pulsedness load". More pragmatically, the differential impact of migration pulsedness on different loci might be used as a detectable signature in genomic data. One can distinguish loci with different selection pressures in the focal population. With a single snapshot of allelic frequencies (or fixation probabilities) at those many different loci across the genome provided, it may be possible to fit a continuous-migration model, and detect the homogenization of fixation rates caused by migration pulsedness, as exemplified in figure 5. Of course, building such a statistical test and evaluating 
its power and applicability is beyond the scope of this article. Still, the approach has the advantage of requiring allele frequency data only, e.g. pool-seq data and Site Frequency Spectrums (SFSs), that are increasingly available for a broad range of organisms. Existing methods to infer migration pulses would typically use individual genome sequences or haplotypes to detect repeated admixture events, and exploit neutral variation only (Marchi et al., 2021). The approach we have sketched here could thus provide a way to improve our methodological arsenal to infer the occurrence of pulsed migration from genomic data.

Our results have direct implications for biological conservation, population management and island biology. For instance, population reinforcement usually take the form of periodic releases of groups of individuals, whose frequency and intensity must both be optimized. Our results suggest that rare introductions of relatively large numbers of individuals, i.e. pulsed introduction patterns, would favor the spread into the focal population of deleterious mutations, and disfavor the establishment of favorable mutations. In short, it is detrimental to population fitness and local adaptation, relative to more continuous fluxes of individuals, possibly compromising population viability and persistence. Of course, this impact would combine with other effects, in particular demographical effects. It has been shown for instance that in small populations subject to Allee effects, pulsed migration patterns can be favourable to population establishment and persistence (Rajakaruna et al., 2013; Bajeux et al., 2019). Similarly, Peniston et al. (2019) analyzed the impact of migration pulsedness for small peripheral populations that are demographic sinks. In the case most similar to our assumptions, Peniston et al. (2019) observed that migration pulsedness could promote local adaptation, in superficial contradiction with our results for strongly deleterious alleles. However, in our case the population was small but viable, whereas in theirs, the sink population was sustained through gene-flow only. This implies that the overall migration rate, i.e. the "mass effect", had to be strong. Since for large values of $m$, we showed that predictions are shifted towards negative effects of pulsedness, the results of Peniston et al. (2019) are in fact consistent with our predictions. These examples underline how complex it is to determine an optimal management policy when demo-genetics effects are taken into account.

Our conclusions extend to broader scenarios as well. For instance, it is relatively easy to apply our approach to the case of two diverging populations, connected by bi-directional migration, and undergoing mutation (not shown in this article). The main conclusions are similar: more pulsed migration patterns would reduce the level of local adaptation and hamper adaptive divergence, while promoting neutral genetic divergence reducing the spread of commonly beneficial mutations. This shows how migration variability may have important consequences for population divergence (e.g. Mailund et al., 2012), and ultimately for the process of speciation (see also Yamaguchi \& Iwasa, 2013). Extending our reasoning to island communities (Cowie \& Holland, 2006), this also suggests that sporadic but potentially intense bouts of immigration, as brought-up by rafting and human-driven invasions, could favour the establishment of relatively maladapted mainland species within communities competing for similar resources.

We conclude by joining Peniston et al. (2019) in saying that it is time to test ex- 
614 isting predictions on migration pulsedness, in empirical systems with well designed 615 experimental set-ups. We add that available data on migration flows, e.g. from 616 monitoring of oceanic rafting, individual tracking of dispersal routes, or marine and 617 aerial current models (Ser-Giacomi et al., 2015; Lagomarsino Oneto et al., 2020), 618 combined with genomics data, may also provide opportunities for testing such pre${ }_{619}$ dictions. Understanding the consequences of ecological variability is increasingly 620 important in the present context of climate and biodiversity change, and the spatio${ }_{621}$ temporal variability in migration flows probably deserves to receive more attention. 


\section{References}

Bajeux, N., Grognard, F. \& Mailleret, L. (2019) Influence of the components of propagule pressure, Allee effects, and stochasticity on the time to establish introduced populations. Journal of Theoretical Biology, 471, $91-107$.

Benestan, L., Fietz, K., Loiseau, N., Guerin, P.E., Trofimenko, E., Rühs, S., Schmidt, C., Rath, W., Biastoch, A., Pérez-Ruzafa, A., Baixauli, P., Forcada, A., Arcas, E., Lenfant, P., Mallol, S., Goñi, R., Velez, L., Höppner, M., Kininmonth, S., Mouillot, D., Puebla, O. \& Manel, S. (2021) Restricted dispersal in a sea of gene flow. Proceedings of the Royal Society B: Biological Sciences, 288, 20210458.

Bishop, L. (1990) Meteorological Aspects of Spider Ballooning. Environmental Entomology, 19, 1381-1387.

Blanquart, F., Kaltz, O., Nuismer, S.L. \& Gandon, S. (2013) A practical guide to measuring local adaptation. Ecology Letters, 16, 1195-1205.

Bobadilla, M. \& Santelices, B. (2005) Variations in the dispersal curves of macroalgal propagules from a source. Journal of Experimental Marine Biology and Ecology, 327, $47-57$.

Boedeltje, G., Bakker, J.P., Ten Brinke, A., Van Groenendael, J.M. \& Soesbergen, M. (2004) Dispersal phenology of hydrochorous plants in relation to discharge, seed release time and buoyancy of seeds: the flood pulse concept supported. Journal of Ecology, $\mathbf{9 2}$, 786-796.

Bürger, R. (2014) A survey of migration-selection models in population genetics. Discrete Continuous Dynamical Systems - B, 19, 883-959.

Bürger, R. \& Akerman, A. (2011) The effects of linkage and gene flow on local adaptation: A two-locus continent-island model. Theoretical Population Biology, 80, 272-288.

Carlton, J.T., Chapman, J.W., Geller, J.B., Miller, J.A., Carlton, D.A., McCuller, M.I., Treneman, N.C., Steves, B.P. \& Ruiz, G.M. (2017) Tsunami-Driven Rafting: Transoceanic Species Dispersal and Implications for Marine Biogeography. Science, 357, $1402-1406$.

Carlton, J. \& Cohen, A. (2003) Episodic Global Dispersal in Shallow Water Marine Organisms: The Case History of the European Shore Crabs Carcinus Maenas and C-Aestuarii. Journal of Biogeography, 30, 1809-1820.

Catalano, K.A., Dedrick, A.G., Stuart, M.R., Puritz, J.B., Montes Jr., H.R. \& Pinsky, M.L. (2020) Quantifying dispersal variability among nearshore marine populations. Molecular Ecology, $\mathbf{n} / \mathbf{a}$.

Cowie, R.H. \& Holland, B.S. (2006) Dispersal is fundamental to biogeography and the evolution of biodiversity on oceanic islands. Journal of Biogeography, 33, 193-198.

Feder, A.F., Pennings, P.S., Hermisson, J. \& Petrov, D.A. (2019) Evolutionary Dynamics in Structured Populations Under Strong Population Genetic Forces. G3: Genes, Genomes, Genetics, 9, 3395-3407. 
Felsenstein, J. (1976) The theoretical population genetics of variable selection and migration. Annual Review of Genetics, 10, 253-280. PMID: 797310.

Folinsbee, K.E. \& Brooks, D.R. (2007) Miocene hominoid biogeography: pulses of dispersal and differentiation. Journal of Biogeography, 34, 383-397.

Gaggiotti, O. \& Smouse, P. (1996) Stochastic Migration and Maintenance of Genetic Variation in Sink Populations. American Naturalist, 147, 919-945.

Garant, D., Forde, S. \& Hendry, A. (2007) The multifarious effects of dispersal and gene flow on contemporary adaptation. Functional Ecology, 21, 434-443.

Garden, C., Currie, K., Fraser, C. \& Waters, J. (2014) Rafting dispersal constrained by an oceanographic boundary. Marine Ecology Progress Series, 501, 297-302.

Goel, N.S. \& Richter-Dyn, N. (1974) Stochastic models in biology. Academic Press., New York, San Francisco, London.

Gomulkiewicz, R., Holt, R.D. \& Barfield, M. (1999) The Effects of Density Dependence and Immigration on Local Adaptation and Niche Evolution in a Black-Hole Sink Environment. Theoretical Population Biology, 55, 283-296.

Hewitt, G. (2000) The Genetic Legacy of the Quaternary Ice Ages. Nature, 405, 907-913.

Johnson, K.P., Adler, F.R. \& Cherry, J.L. (2000) Genetic and phylogenetic consequences of island biogeography. Evolution, 54, 387-396.

Keyse, J., Treml, E.A., Huelsken, T., Barber, P.H., DeBoer, T., Kochzius, M., Nuryanto, A., Gardner, J.P.A., Liu, L.L., Penny, S. \& Riginos, C. (2018) Historical divergences associated with intermittent land bridges overshadow isolation by larval dispersal in co-distributed species of Tridacna giant clams. Journal of Biogeography, 45, 848-858.

Kimura, M. (1962) On the probability of fixation of mutant genes in a population. Genetics, 47, 713-719.

Kimura, M. \& Ohta, T. (1969) The average number of generations until fixation of a mutant gene in a finite population. Genetics, 61, 763-771.

Kobayashi, Y., Hammerstein, P. \& Telschow, A. (2008) The neutral effective migration rate in a mainland-island context. Theoretical Population Biology, 74, $84-92$.

Lagomarsino Oneto, D., Golan, J., Mazzino, A., Pringle, A. \& Seminara, A. (2020) Timing of fungal spore release dictates survival during atmospheric transport. Proceedings of the National Academy of Sciences, 117, 5134-5143.

Lande, R. (1994) Risk of population extinction from fixation of new deleterious mutations. Evolution, 48, 1460-1469.

Latter, B.D.H. \& Sved, J.A. (1981) Migration and Mutation in Stochastic Models of Gene Frequency Change. II. Stochastic Migration with a Finite Number of Islands. J. Math. Biology, 13, 95-104.

Lenormand, T. (2002) Gene Flow and the Limits to Natural Selection. Trends in Ecology and Evolution, 17, 183-189. 
Mailund, T., Halager, A.E., Westergaard, M., Dutheil, J.Y., Munch, K., Andersen, L.N., Lunter, G., Prüfer, K., Scally, A., Hobolth, A. \& Schierup, M.H. (2012) A New Isolation with Migration Model along Complete Genomes Infers Very Different Divergence Processes among Closely Related Great Ape Species. PLOS Genetics, 8, 1-19.

Manna, F., Martin, G. \& Lenormand, T. (2011) Fitness Landscapes: An Alternative Theory for the Dominance of Mutation. Genetics, 189, 923-937.

Marchi, N., Schlichta, F. \& Excoffier, L. (2021) Demographic inference. Current Biology, 31, R276-R279.

Martin, C.H. \& Turner, B.J. (2018) Long-distance dispersal over land by fishes: extremely rare ecological events become probable over millennial timescales. Proceedings of the Royal Society B: Biological Sciences, 285, 20172436.

Maruyama, T. (1970) Effective number of alleles in a subdivided population. Theoretical Population Biology, 1, 273-306.

Masson-Delmotte, V., Zhai, P., Pörtner, H.O., Roberts, D., Skea, J., Shukla, P., Pirani, A., Moufouma-Okia, W., Péan, C., Pidcock, R., Connors, S., Matthews, J., Chen, Y., Zhou, X., Gomis, M., Lonnoy, E., Maycock, T., Tignor, M. \& Waterfield, T. (2018) IPCC, 2018: Global Warming of $1.5^{\circ} \mathrm{C}$. An IPCC Special Report on the impacts of global warming of $1.5^{\circ} \mathrm{C}$ above pre-industrial levels ad related global greenhouse gas emission pathways, in the context of strengthening the global response to the threat of climate change, sustainable development, and efforts to eradicate poverty. Working Group I Technical Support Unit, Bern, Switzerland.

Matias, M.G., Mouquet, N. \& Chase, J.M. (2013) Dispersal stochasticity mediates species richness in sourcesink metacommunities. OIKOS, 122, 395-402.

Maynard-Smith, J. (1966) Sympatric Speciation. The American Naturalist, 100, 637-650.

Mills, L.S. \& Allendorf, F.W. (1996) The One-Migrant-per-Generation Rule in Conservation and Management. Conservation Biology, 10, 1509-1518.

Moran, P.A.P. (1962) The statistical processes of evolutionary theory. Clarendon Press; Oxford University Press.

Morris-Pocock, J.A., Anderson, D.J. \& Friesen, V.L. (2016) Biogeographical barriers to dispersal and rare gene flow shape population genetic structure in red-footed boobies (Sula sula). Journal of Biogeography, 43, 2125-2135.

Nagylaki, T. (1979) The Island Model with Stochastic Migration. Genetics, 91, 163-176.

Otto, S.P. \& Whitlock, M.C. (2013) Fixation probabilities and times. eLS. American Cancer Society. ISBN 978-0-470-01590-2.

Palstra, F.P. \& Ruzzante, D.E. (2008) Genetic estimates of contemporary effective population size: what can they tell us about the importance of genetic stochasticity for wild population persistence? Molecular Ecology, 17, 3428-3447.

Peacock, M.M. \& Smith, A.T. (1997) The effect of habitat fragmentation on dispersal patterns, mating behavior, and genetic variation in a pika (Ochotona princeps) metapopulation. Oecologia, 112, 524-533. 
Peniston, J.H., Barfield, M. \& Holt, R.D. (2019) Pulsed Immigration Events Can Facilitate Adaptation to Harsh Sink Environments. The American Naturalist, 194, 316-333.

Rajakaruna, H., Potapov, A. \& Lewis, M. (2013) Impact of stochasticity in immigration and reintroduction on colonizing and extirpating populations. Theoretical Population Biology, 85, 38-48.

Reed, D.C., Laur, D.R. \& Ebeling, A.W. (1988) Variation in Algal Dispersal and Recruitment: The Importance of Episodic Events. Ecological Monographs, 58, 321-335.

Reiners, W.A. \& Driese, K.L. (2004) Transport Processes in Nature: Propagation of Ecological Influences Through Environmental Space, vol. 2. Cambridge University Press.

Renner, S. (2004) Plant Dispersal across the Tropical Atlantic by Wind and Sea Currents. International Journal of Plant Sciences, 165, S23-S33.

Rice, S.H. \& Papadopoulos, A. (2009) Evolution with Stochastic Fitness and Stochastic Migration. PLOS ONE, 4, 1-12.

Rousset, F. (2013) Genetic structure and selection in subdivided populations (MPB-40), vol. 40. Princeton University Press.

Ser-Giacomi, E., Rossi, V., López, C. \& Hernández-García, E. (2015) Flow networks: A characterization of geophysical fluid transport. Chaos: An Interdisciplinary Journal of Nonlinear Science, 25, 036404.

Slatkin, M. (1981) Fixation Probabilities and Fixation Times in a Subdivided Population. Evolution, 35, 477-488.

Smith, T.M., York, P.H., Broitman, B.R., Thiel, M., Hays, G.C., van Sebille, E., Putman, N.F., Macreadie, P.I. \& Sherman, C.D.H. (2018) Rare long-distance dispersal of a marine angiosperm across the Pacific Ocean. Global Ecology and Biogeography, 27, 487-496.

Soubeyrand, S., Sache, I., Hamelin, F. \& Klein, E.K. (2015) Evolution of dispersal in asexual populations: to be independent, clumped or grouped? Evolutionary Ecology, 29, 947-963.

Thurman, T.J. \& Barrett, R.D.H. (2016) The genetic consequences of selection in natural populations. Molecular Ecology, 25, 1429-1448.

Tigano, A. \& Friesen, V.L. (2016) Genomics of local adaptation with gene flow. Molecular Ecology, 25, 2144-2164.

Wang, J. \& Whitlock, M.C. (2003) Estimating Effective Population Size and Migration Rates From Genetic Samples Over Space and Time. Genetics, 163, 429-446.

White, C., Selkoe, K.A., Watson, J., Siegel, D.A., Zacherl, D.C. \& Toonen, R.J. (2010) Ocean currents help explain population genetic structure. Proceedings of the Royal Society B: Biological Sciences, 277, 1685-1694.

Whitlock, M.C. (1992) Temporal fluctuations in demographic parameters and the genetic variance among populations. Evolution, 46, 608-615. 
Whitlock, M.C. (2003) Fixation Probability and Time in Subdivided Populations. Genetics, 164, 767-779.

778 Wright, S. (1931) Evolution in Mendelian Populations. Genetics, 16, 97-159.

Yamaguchi, R. \& Iwasa, Y. (2013) First Passage Time to Allopatric Speciation. Interface focus, 3, 20130026.

781

Yamazaki, Y., Naoe, S., Masaki, T. \& Isagi, Y. (2016) Temporal variations in seed dispersal patterns of a bird-dispersed tree, Swida controversa (Cornaceae), in a temperate forest.

783 Ecological Research, 31, 165-176.

Yeaman, S. \& Otto, S.P. (2011) Establishment and Maintenance of Adaptive Genetic Divergence under Migration, Selection, and Drift. Evolution, 65, 2123-2129. 